\title{
Routing in the Dark: Pitch Black
}

\author{
Nathan S. Evans \\ Chris GauthierDickey \\ Christian Grothoff
Colorado Research Institute
for Security and Privacy \\ Department of Computer Science \\ University of Denver, USA \\ \{natevans,chrisg,grothoff $\} @$ cs.du.edu
}

\begin{abstract}
In many networks, such as mobile ad-hoc networks and friend-to-friend overlay networks, direct communication between nodes is limited to specific neighbors. Often these networks have a small-world topology; while short paths exist between any pair of nodes in small-world networks, it is non-trivial to determine such paths with a distributed algorithm. Recently, Clarke and Sandberg proposed the first decentralized routing algorithm that achieves efficient routing in such small-world networks.

This paper is the first independent security analysis of Clarke and Sandberg's routing algorithm. We show that a relatively weak participating adversary can render the overlay ineffective without being detected, resulting in significant data loss due to the resulting load imbalance. We have measured the impact of the attack in a testbed of 800 nodes using minor modifications to Clarke and Sandberg's implementation of their routing algorithm in Freenet. Our experiments show that the attack is highly effective, allowing a small number of malicious nodes to cause rapid loss of data on the entire network.

We also discuss various proposed countermeasures designed to detect, thwart or limit the attack. While we were unable to find effective countermeasures, we hope that the presented analysis will be a first step towards the design of secure distributed routing algorithms for restricted-route topologies.
\end{abstract}

\section{Introduction}

Fully decentralized and efficient routing algorithms for restricted route networks promise to solve crucial problems for a wide variety of networking applications. Efficient decentralized routing is important for sensor and general wireless networks, peer-to-peer overlay networks and theoretically even next generation Internet (IP) routing. A number of distributed peer-to-peer routing protocols developed in recent years achieve scalable and efficient routing by constructing a structured overlay topology $[4,5,8,11,15]$. However, all of these designs are unable to work in realworld networks with restricted routes. In a restricted route topology, nodes can only directly communicate with a subset of other nodes in the network. Such restrictions arise from a variety of sources, such as physical limitations of the communications infrastructure (wireless signals, physical network topology), policies (firewalls) or limitations of underlying protocols (NAT, IPv6-IPv4 interaction).

Recently, a new routing algorithm for restricted route topologies was proposed [13] and implemented in version 0.7 of Freenet, an anonymous peer-to-peer file-sharing network [3]. The proposed algorithm achieves routing in expected $O(\log n)$ hops for small-world networks with $n$ nodes and $O(\log n)$ neighbors ${ }^{1}$ by having nodes swap $l o$ cations in the overlay under certain conditions. This significant achievement raises the question of whether the algorithm is robust enough to become the foundation for the large domain of routing in restricted route networks.

The research presented in this paper shows that any participating node can severely degrade the performance of the routing algorithm by changing the way it participates in the location swapping aspect of the protocol. Most of

\footnotetext{
${ }^{1}$ Given only a constant number of neighbors, the routing cost increases to $O\left(\log ^{2} n\right)$.
} 
the guards in the existing routing implementation are ineffective or severely limited and in particular fail to reliably detect the malicious nodes. Experiments using a Freenet testbed show that a small fraction of malicious nodes can dramatically degenerate routing performance and cause massive content loss in a short period of time. Our research also illuminates why churn impacts the structure of the overlay negatively, a phenomenon that was observed by Freenet users in practice but has, to the best of our knowledge, never been explained.

The paper is structured as follows. Section 2 describes related work focusing on distributed hash tables and smallworld networks. Section 3 details Freenet's distributed friend-to-friend (or, as termed by the Freenet authors, "darknet") routing algorithm for small-world networks. The proposed attack is described in Section 4, followed by experimental results showing the effects of the attack in Section 5. Possible defenses and their limitations are discussed in Section 6.

\section{Related Work}

\subsection{Distributed hash tables}

A distributed hash table is a data structure that enables efficient key-based lookup of data in a peer-to-peer overlay network. Generally, the participating peers maintain connections to a relatively small subset of the other participants in the overlay. Each peer is responsible for storing a subset of the key-value pairs and for routing requests to other peers. In other words, a key property of the use of DHTs in a peer-to-peer setting is the need to route queries in a network over multiple hops based on limited knowledge about which peers exist in the overlay network. Part of the DHT protocol definition is thus concerned with maintaining the structure of the network as peers join or leave the overlay.

DHT designs can be characterized using the performance metrics given in Table 1. Routing in DHTs is generally done in a greedy fashion and resembles lookups in skip lists [10]. Table 2 summarizes the key properties of various existing DHT designs. The table does not capture properties which are hard to quantify, such as fault-tolerance. Given a uniform distribution of keys, most existing DHT designs achieve near perfect load balancing between peers. Hosts that can provide significantly more resources than others are usually accommodated by associating multiple locations in the overlay with a single host. In some sense, those hosts are counted as multiple peers.

A major limitation of the DHT designs listed in Table 2 is that they do not support routing in restricted route topologies. These DHTs assume that it is generally possible for any peer to connect to any other peer. However, firewalls and network address translation (NAT) make this assumption unrealistic over the current Internet, where large- scale studies have shown that over $70 \%$ of machines are NATed [1].

In contrast to the DHT designs from Table 2, the Freenet routing algorithm achieves expected $O(\log n)$ routing in restricted route topologies under the assumption that the restricted network topology has small-world properties.

\subsection{Small-world networks}

A small-world network is informally defined as a network where the average shortest path between any two nodes is "small" compared to the size of the network, where "small" is generally considered to mean at least logarithmic in relation to the size of the network. Small world networks occur frequently in the real world [17], the most prominent example being social networks [9].

Watts and Strogatz [17] characterized small-world networks as an intermediate stage between completely structured networks and random networks. According to their definition, small world networks with $n$ nodes have on average $k$ edges per vertex where $n>>>>\log n$. They define a clustering coefficient which captures the amount of structure (clustering) in a given network. Small-world networks are then networks with a clustering coefficient significantly larger than the coefficients of completely random networks and with average shortest path lengths close to those of completely random networks. Watts and Strogatz's work explains why short paths exist in real-world networks.

Kleinberg [6, 7] generalized Watts and Strogatz' construction of small-world networks and gave sufficient and necessary conditions for the existence of efficient distributed routing algorithms for these constructions. Kleinberg's model for distributed routing algorithms does not include the possibility of nodes swapping locations, which is a fundamental part of Freenet's "darknet" routing algorithm.

\section{Freenet's "darknet" routing algorithm}

Freenet [3] is a peer-to-peer network where the operator of each node specifies which other peers are allowed to connect to the node [2]. The main reason for this is to obscure the participation of a node in the network - each node is only directly visible to the friends of its' operator. Peer-topeer networks that limit connections to friend-to-friend interactions are sometimes called darknets. Given that social networks are small-world networks and that small-world networks arise easily given a certain amount of "randomness" in the graph construction, it is realistic to assume that Freenet's darknet is a small-world network. The routing restrictions imposed on the Freenet overlay could technically model arbitrary network limitations; consequently, an efficient distributed routing algorithm for such a topology should easily generalize to any small-world network. 
(1) Messages required for each key lookup

(2) Messages required for each store operation

(3) Messages needed to integrate a new peer

(4) Messages needed to manage a peer leaving

(5) Number of connections maintained per peer

(6) Topology can be adjusted to minimize per-hop latency (yes/no)

(7) Connections are symmetric or asymmetric

Table 1. Performance metrics for DHTs.

\begin{tabular}{c||c|c|c|c|c} 
& Chord [15] & Pastry [12] & Kademlia [8] & CAN [11] & RSG [5] \\
\hline \hline$(1)$ & $O(\log n)$ & $O(\log n)$ & $O(\log n)$ & $O\left(n^{-d}\right)$ & $O(\log n)$ \\
\hline$(2)$ & $O(\log n)$ & $O(\log n)$ & $O(\log n)$ & $O\left(n^{-d}\right)$ & $O(\log n)$ \\
\hline$(3)$ & $O\left(\log ^{2} n\right)$ & $O(\log n)$ & $O(\log n)$ & $O\left(d+n^{-d}\right)$ & $O(\log n)$ \\
\hline$(4)$ & $O\left(\log ^{2} n\right)$ & $O(1)$ & $O(1)$ & $O(d)$ & $O(\log n)$ \\
\hline$(5)$ & $O(\log n)$ & $O(\log n)$ & $O(\log n)$ & $O(d)$ & $O(1)$ \\
\hline$(6)$ & no & yes & yes & yes & no \\
\hline (7) & asymmetric & asymmetric & symmetric & symmetric & asymmetric
\end{tabular}

\section{Table 2. Comparison of DHT designs. The numbers refer to the list of performance metrics given in Table 1. The value $d$ is a system parameter for CAN.}

\subsection{Network creation}

The graph of the Freenet network consists of vertices, which are peers, and edges, which are created by friend relationships. An edge only exists between peers if both operators have agreed to the connection a priori. Freenet assumes that a sufficient number of edges (or friend relationships) between peers will exist so that the network will be connected.

Each Freenet node is created with a unique, immutable identifier and a randomly generated initial location. The identifier is used by operators to specify which connections are allowed, while the location is used for routing. The location space has a range of $[0,1)$ and is cyclic with 0 and 1 being the same point. For example, the distance between nodes at locations 0.1 and 0.9 is 0.2 .

Data stored in the Freenet network is associated with a specific key from the range of the location space. The routing algorithm transmits get and put requests from node $A$ to the neighbors of $A$ starting with the neighbor with the closest location to the key of the request.

\subsection{Operational overview}

The basic strategy of the routing algorithm is to greedily forward a request to the neighbor whose location is closest to the key. However, the simple greedy forwarding is not guaranteed to find the closest peer - initially, the lo- cation of each peer is completely random and connections between peers are restricted (since a peer can only establish connections to other peers which the operator has explicitly allowed). Consequently, the basic greedy algorithm is extended to a depth-first search of the topology (with bounded depth) where the order of the traversal is determined by the distance of the nodes to the key [14]. Figure 1 shows the routing algorithm for get operations in pseudocode. A put operation is routed in the same fashion and reaches exactly the same peers as an unsuccessful get operation. In addition, Freenet replicates content transmitted as part of a get response or as part of a put operation at nodes that are encountered during the routing process where the node's location is closer to the key than the location of any of the peer's neighbors.

Both get and put requests include a hops-to-live value which is initially set to the nodes pre-set maximum and used to limit traversal of the network. Each request also includes the closest location (in relation to the key) of any node encountered so far during the routing process.

\subsection{Location swapping}

To make the routing algorithm find the data faster, Freenet attempts to cluster nodes with similar locations. Let $L(n)$ denote the current location of node $n$. The network achieves this by having nodes periodically consider swapping their locations using the following algorithm: 
1. A node $A$ randomly chooses a node $B$ in its proximity and initiates a swap request. Both nodes share the locations of their respective neighbors and calculate $D_{1}(A, B) . D_{1}(A, B)$ is the product of the existing distances between $A$ and each of $A$ 's neighbors $|L(a)-L(n)|$ multiplied by the product of the existing distances between $B$ and each of $B$ 's neighbors.

$$
D_{1}(A, B)=\prod_{(A, n) \in E}|L(A)-L(n)| \cdot \prod_{(B, n) \in E}|L(B)-L(n)|
$$

2. The nodes also compute $D_{2}(A, B)$, the product of the products of the differences between their locations and their neighbors' locations after a potential swap:

$$
D_{2}(A, B)=\prod_{(A, n) \in E}|L(B)-L(n)| \cdot \prod_{(B, n) \in E}|L(A)-L(n)|
$$

3. If the nodes find that $D_{2}(A, B) \leq D_{1}(A, B)$, they swap locations, otherwise they swap locations with probability $\frac{D_{1}(A, B)}{D_{2}(A, B)}$. The deterministic swap always decreases the average distances of nodes with their neighbors. The probabilistic swap is used to escape local minima.

The overlay becomes semi-structured as a result of swapping locations; the routing algorithm's depth first search can

\section{Figure 1. Pseudocode for routing of a get re- quest.}

1. Check that the new get request is not identical to recently processed requests; if the request is a duplicate, notify sender about duplication status, otherwise continue.

2. Check local data store for the data; if the data is found, send response to sender, otherwise continue.

3 . If the current location is closer to the key than any previously visited location, reset hops-to-live to the maximum value.

4. If hops-to-live of the request is zero, respond with data not found, otherwise continue.

5. Find the closest neighbor (in terms of peer location) with respect to the key of the get request, excluding those routed to already. Forward the request to the closest peer with a (probabilistically) decremented hops-to-live counter. If valid content is found, forward the content to sender, otherwise, repeat step 5. utilize this structure in order to find short paths with high probability. Sandberg's thesis [13] shows that the Freenet routing algorithm converges towards routing in $O(\log n)$ steps (with high probability) under the assumption that the set of legal connections specified by the node operators forms a small-world network. This is a significant result because it describes the first fully decentralized distributed hash table (DHT) design that achieves $O(\log n)$ routing with (severely) restricted routes. Most other DHT designs make the unrealistic assumption that every node is able to directly communicate with every other node $[5,8,12,15]$.

\subsection{Content Storage}

Each Freenet node stores content in a datastore of bounded size. Freenet uses a least-recently-used content replacement policy, removing the least-recently-used content when necessary to keep the size of the datastore below the user-specified limit.

Our simulation of routing in the Freenet network places the content at the node whose location is closest to the key and does not allow caching or replication of content. The reason for this is that our study focuses on routing performance and not on content replication and caching strategies.

\subsection{Example}

Figure 2 shows a small example network. Each node is labeled with its location $\left(L_{n} \in[0,1)\right)$ in the network. The bi-directional edges indicate the direct connections between nodes. In a friend-to-friend network, these are the connections that were specifically allowed by the individual node operators, and each node is only aware of its immediate neighbors. Similarly, in an ad-hoc wireless network, the edges would indicate which nodes could physically communicate with each other. While our example network lacks cycles, any connected graph is allowed; the small-world property is only required to achieve $O(\log n)$ routing performance as the algorithm works for any connected graph.



Figure 2. This figure shows an example network with two nodes considering a swap. The result of the swap equation is $D_{1}=.60$

* $.65 * .25 * .50=.04875$ and $D_{2}=.30 * .35 * .05$

${ }^{\star} .80=.0042$. Since $D_{1}>D_{2}$, they swap. 


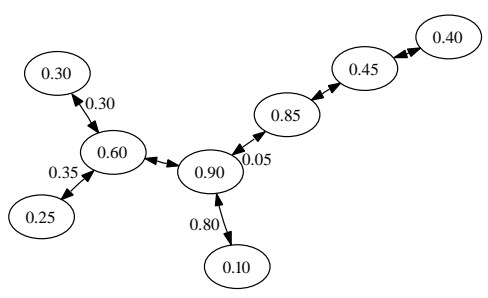

Figure 3. This figure shows the resulting example network after the swap has occurred.

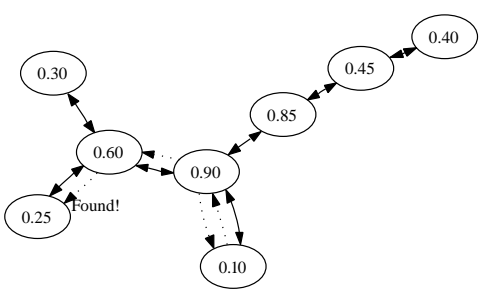

Figure 4. Illustrates the path of a GET request initiated from the node with location of 0.90 . The request is looking for data with a key value of .23 , which is stored at the node identified by the location 0.25 . The path that the GET request travels is displayed as the dotted lines which travel from $0.90 \rightarrow 0.10 \rightarrow 0.90$ $\rightarrow 0.60 \rightarrow 0.25$ where the data is found.

The network illustrated in Figure 2 happens to have an assignment of locations that would cause the nodes with locations 0.60 and 0.90 to perform a swap in order to minimize the distance product from Equation (1). Figure 3 shows the new assignment of locations after the swap. Note that after a swap each node retains exactly the same set of connections; the only change is in the location identifiers of the nodes. This change in node locations impacts the order of the traversal during routing.

Figure 4 shows how a GET request would be routed after the swap (with a maximum value of hops-to-live larger or equal to two). Starting at the node with location 0.90 and targeting the key 0.23 the node picks its closest neighbor (with respect to the key), which is 0.10 . However, 0.10 does not have the content and also lacks other neighbors to route to and thus responds with content not found. Then 0.90 attempts its' second-closest neighbor, 0.60. Again, 0.60 does not have the content, but it has other neighbors. The 0.25 neighbor is closest to 0.23 . The content is found at that node and returned via 0.60 (the restricted-route topology does not allow 0.25 to send the result directly back to 0.90 ).

Finally, Figure 5 illustrates how Freenet routes a PUT request with a maximum value of 1 for hops-to-live (in prac- tice, the maximum value would be bigger). The algorithm again attempts to find the node with the closest location in a greedy fashion. Once a node $C$ is found where all neighbors are further away from the node, the neighbors fail to reset hops-to-live (since 0.90 is closer to the key than they are), which ends the process.

\section{Security Analysis}

The routing algorithm works under the assumption that the distribution of the keys and peer locations is random. In that case, the load is balanced. In particular, all nodes are expected to store roughly the same amount of content and all nodes are expected to receive roughly an equivalent numbers of requests.

The basic idea behind the attack is to de-randomize the distribution of the node locations. The attacker tries to cluster the locations around a particular small set of values.
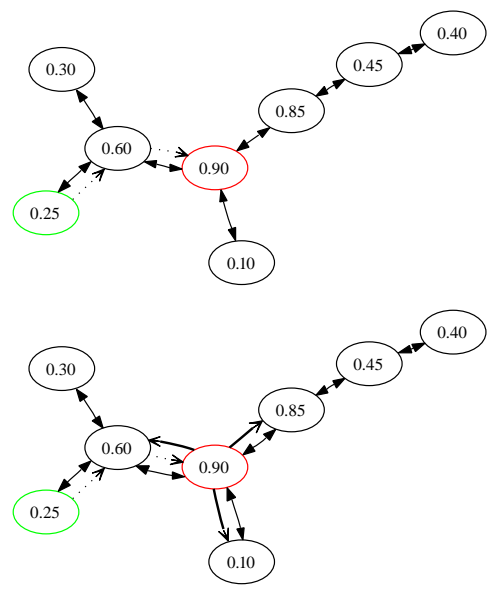

Figure 5. The graph on top illustrates the path of a PUT request inserting data with a key of .96. The request is initiated from node with location 0.25 . The path that the PUT request travels is displayed as the dotted lines which travel from $0.25 \rightarrow 0.60 \rightarrow 0.90$, where the data is stored. The bottom graph shows what happens after a PUT has found a node whose neighbors are all further away from the key. The node 0.90 (as all of the predecessors on the path) resets the hops-to-live value to its maximum (in this case, one) and forwards the PUT request to all of its neighbors. Since these neighbors are not closer to the key than their predecessor they do not reset hops-to-live. Since the value reaches zero, routing ends. 
Since the distribution of the keys is still random and independent of the distribution of the node locations, the clustering of node locations around particular values results in an uneven load distribution. Nodes within the clusters are responsible for less content (because many other nodes are also close to the same set of keys), whereas the load for nodes outside of the clusters is disproportionately high.

We will now detail two scenarios which destroy the initial random distribution of node locations resulting in the clustering of locations around particular values. The first scenario uses attack nodes inside the network. This attack quickly unbalances the load in the network, causing significant data loss; the reason for the data loss is that the imbalance causes some nodes to greatly exceed their storage capacity, whereas other nodes store nothing. The second scenario illustrates how location imbalance can occur naturally even without an adversary due to churn.

\subsection{Active Attack}

As described in Section 3.3, a Freenet node attempts to swap with random peers periodically. Suppose that an attacker wants to bias the location distribution towards a particular location, $m$. In order to facilitate the attack, the attacker assumes that particular location (sets its location to $m$ ). This malicious behavior cannot be detected by the node's neighbors because the attacker can claim to have obtained this location from swapping. A neighbor cannot verify whether such a swap has occurred because the friend-tofriend $(\mathrm{F} 2 \mathrm{~F})$ topology restricts communication to immediate neighbors.

Suppose an attacker node $A$ intends to force a swap with a victim $N$ so that $L(N)=m$ afterwards. Let $N$ have $k$ neighbors. Then $A$ will initiate a swap request with $N$ claiming to have at least $k+1$ neighbors with locations favoring a swap according to Equation (1). Specifically, the locations of the neighbors should be either close to $L(N)$ or close to the maximum distance from $L(A)=m$. The attacker then creates swap requests in accordance with the Freenet protocol. Again, the F2F topology prevents the neighbor involved in the swap from checking the validity of this information. After the swap, the attack node can again assume the original location $m$ and continue to try to swap with its other neighbors whose locations are still random.

The neighbors that have swapped with an attacker then continue to swap in accordance with the swapping algorithm, possibly spreading the malicious locations. Once the location has been spread, the adversary subjects another neighbor to a swap, removing yet another random location from the network. Figure 6 illustrates the impact of a malicious node on the example network after a few swaps (with the attacker using $m \approx 0.5$ ). The likelihood of neighbors spreading the malicious location by swapping can be improved by using multiple attack locations. Thus, a tradeoff exists between the speed of penetration and the impact of the attack in terms of causing load imbalances.

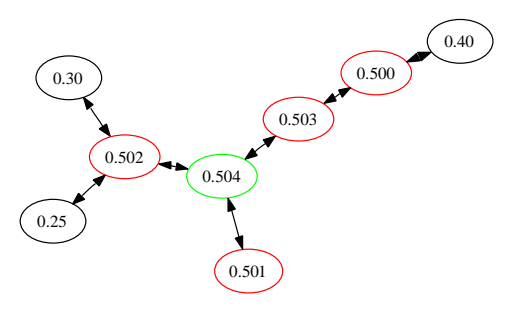

\subsection{Natural Churn}

Network churn, the joining and leaving of nodes in the network, is a crucial issue that any peer-to-peer routing protocol needs to address. We have ignored churn until now because the attack described in the previous section does not require it. Intuition may suggest that natural churn may help the network against the attack by supplying a constant influx of fresh, truly random locations. This section illustrates that the opposite is the case: natural churn can strengthen the attack and even degenerate the Freenet network in the same manner without the presence of malicious nodes.

For the purpose of this discussion, we need to distinguish two types of churn. The first kind, leave-join churn, describes fluctuations in peer availability due to a peer leaving the network for a while and then joining again. In this case, the network has to cope with a temporary loss of availability in terms of connectivity and access to content stored at the node. Freenet's use of content replication and its routing algorithm are well-suited to handle this type of churn. Most importantly, a node leaving does not immediately trigger significant changes at any other node. As a result, an adversary cannot use leave-join churn to disrupt network operations. Since honest Freenet peers re-join with the same location that they last had when they left the network, leave-join churn does not impact the overall distribution of locations in the network.

The second kind, join-leave churn, describes peers who join the network and then leave for good. In this case, the network has to cope with the permanent loss of data stored at this peer. In the absence of adversaries, join-leave churn may be less common in peer-to-peer networks; however, it is always possible for users to discontinue using a particular application. Also, often users may just test an application once and decide that it does not meet their needs. Again, we believe that Freenet's content replication will likely avoid 
significant loss of content due to realistic amounts of joinleave churn.

However, natural join-leave churn has another, rather unexpected impact on the distribution of locations in the Freenet overlay. This additional impact requires that the overlay has a stable core of peers that are highly available and strongly interconnected, which is a common phenomenon in most peer-to-peer networks. In contrast to this set of stable core-peers, peers that contribute to join-leave churn are likely to participate only briefly and have relatively few connections. Suppose the locations $\gamma_{i}$ of the core-peers are initially biased towards (or clustered around) a location $\alpha \in[0,1)$. Furthermore, suppose that (over time) thousands of peers with few connections (located at the fringe of the network) contribute to join-leave churn.

Each of these fringe-peers will initially assign itself a random location $\beta \in[0,1)$. In some cases, this random choice $\beta$ will be closer to $\alpha$ than some of the $\gamma_{i}$-locations of the core nodes. In that case, the routing algorithm is likely to swap locations between these fringe-peers and core-peers in order to reduce the overall distances to neighbors (as calculated according to Equation (1)). Peers in the core have neighbors close to $\alpha$, so exchanging one of the $\gamma_{i}$ 's for $\beta$ will reduce their overall distances. The fringe peers are likely to have few connections to the core group and thus the overall product after a swap is likely to decrease.

Consequently, non-adversarial join-leave churn strengthens any existing bias in the distribution of locations among the long-lived peers. The long-term results of join-leave churn are equivalent to what the attack from Section 4.1 is able to produce quickly - most peers end up with locations clustering around a few values. Note that this phenomenon has been observed by Freenet users and was reported to the Freenet developers - who so far have failed to explain the cause of this degeneration. ${ }^{2}$ Since both the attack and natural churn have essentially the same implications for the routing algorithm, the performance implications established by our experimental results (Section 5) hold for both scenarios.

\section{Experimental Results}

This section presents experimental results obtained from a Freenet testbed with up to 800 active nodes. The testbed, consisting of up to $18 \mathrm{GNU} /$ Linux machines, runs the actual Freenet 0.7 code. The nodes are connected to form a smallworld topology (using Kleinberg's 2d-torus model [6]) with on average $O\left(\log ^{2} n\right)$ connections per node.

Each experiment consists of a number of iterations. In each iteration, nodes are given a fixed amount of time to swap locations. Then the performance of the network is

\footnotetext{
${ }^{2}$ https: //bugs.freenetproject.org/view.php?id=647, April 2007. We suspect that the clustering around 0.0 is caused by software bugs, resulting in an initial bias for this particular value, which is then strengthened by churn.
}

evaluated. The main performance metrics are the average path length needed to find the node that is responsible for a particular key, and the percentage of the content originally available in the network that can still be retrieved.

All nodes are configured with the same amount of storage space. Before each experiment, the network is seeded with content with a random key distribution. The amount of content is fixed at a quarter of the storage capacity of the entire network. The content is always (initially and after each iteration) placed at the node with the closest location to the key. Nodes discard content if they do not have sufficient space. Discarded content is lost for the duration of the experiment.

Depending on the goals of the experiment, certain nodes are switched into attack mode starting at a particular iteration. The attacking nodes are randomly chosen, and behave exactly as all of the other nodes, except for aggressively propagating malicious node locations when swapping.

\subsection{Distribution of Node Locations}

Figures 7 and 8 illustrates the distribution of node locations on a circle before and after an attack. The initial distribution in Figure 7 consists of 800 randomly chosen locations, which are largely evenly distributed over the entire interval.

The distribution shown in Figure 8 illustrates the effect of two nodes attacking the network in an attempt to create eight clusters. Note that the number of attackers and the number of cluster locations can be chosen independently.

Both plots use thicker dots in order to highlight spots where many peers are in close proximity. Particularly after the attack peers often have locations that are so close to each other (at the order of $2^{-30}$ ) that a simple plot of the individual locations would just show a single dot. Thicker dots illustrate the number of peers in close proximity, the spread of their locations is actually much smaller than the thickness may suggest.

\subsection{Availability of Content}

Figures 9, 10 and 11 show the data loss in a simulated Freenet network with 800 nodes and two, four and eight attackers respectively. The attackers attempt to use swapping in order to cluster the locations of nodes in the network around eight pre-determined values. The resulting clustering of many nodes around particular locations causes the remaining nodes to be responsible for disproportionately large areas in the key space. If this content assignment requires a particular node to store more content than the node has space available for, content is lost. 


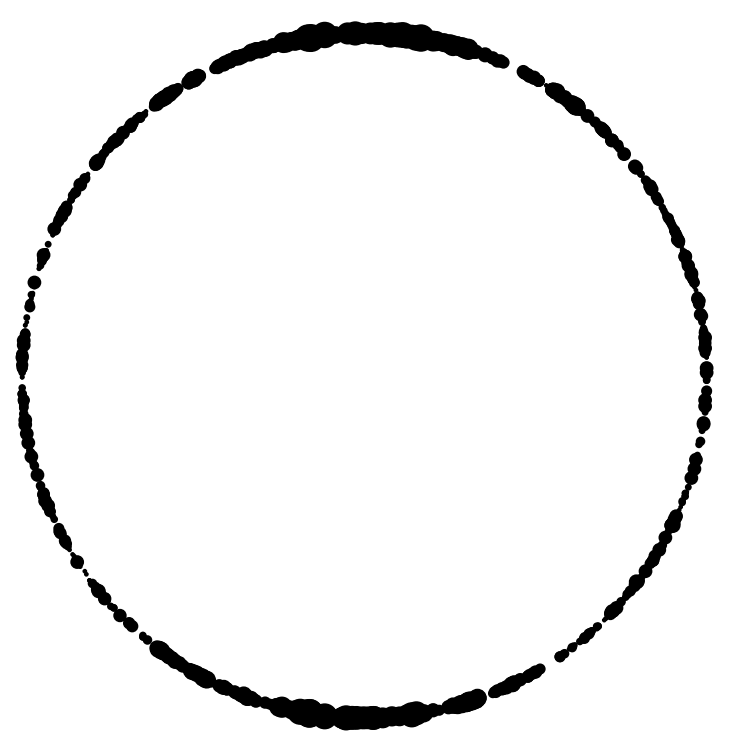

Figure 7. Plot of 800 initial node locations before the attack. Plot points increase in diameter as the density of peers nearby to that location increases.

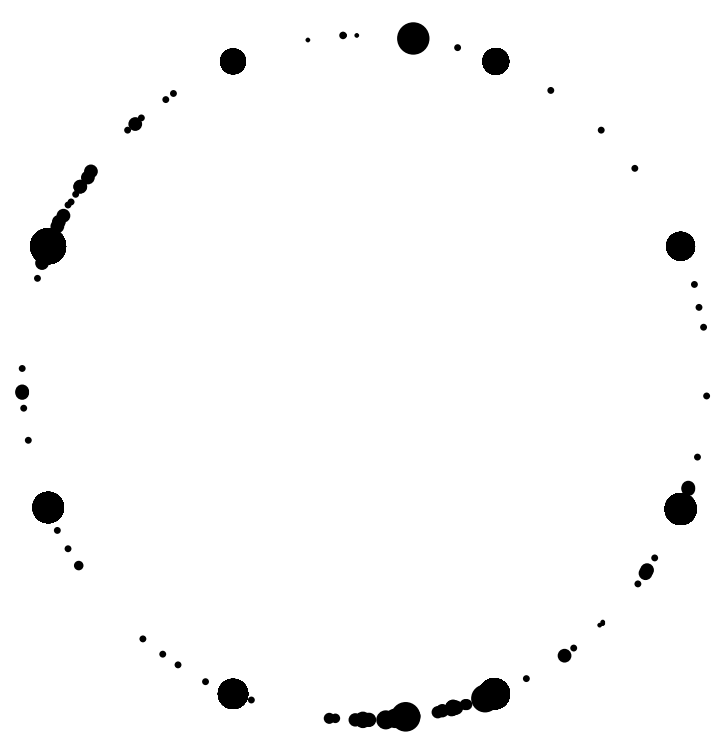

Figure 8. Plot of 800 node locations after the attack by 2 malicious nodes. Plot points increase in diameter as the density of peers nearby to that location increases. The large plot points indicate the success of the attack in clustering most nodes around the 8 chosen locations.

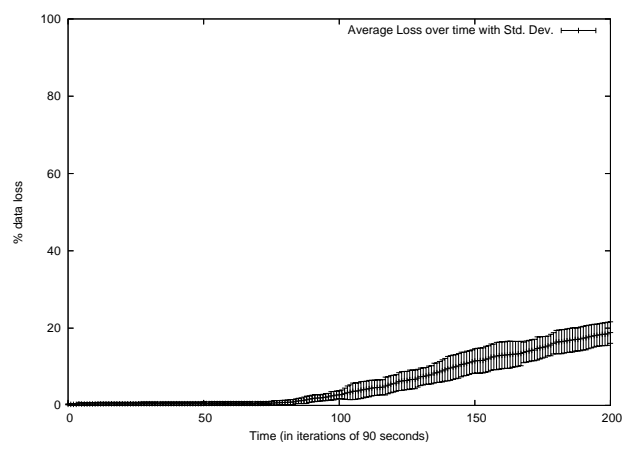

Figure 9. Graph showing average data loss over 5 runs with 800 total nodes and 2 attack nodes using 8 bad locations with the attack starting after about $2 \mathrm{~h}$.

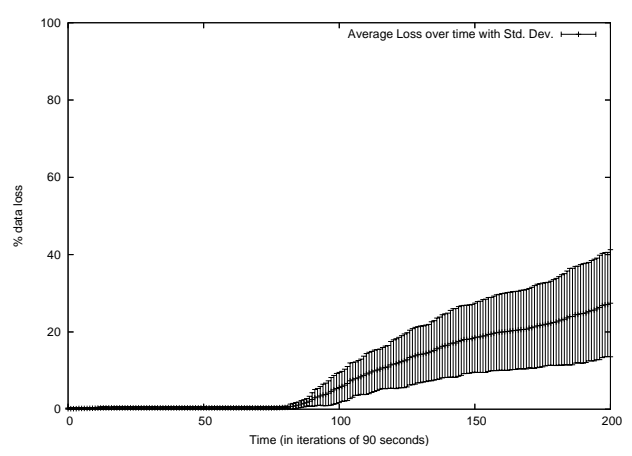

Figure 10. Graph showing average data loss over 5 runs with 800 total nodes and 4 attack nodes using 8 bad locations with the attack starting after about $2 \mathrm{~h}$.

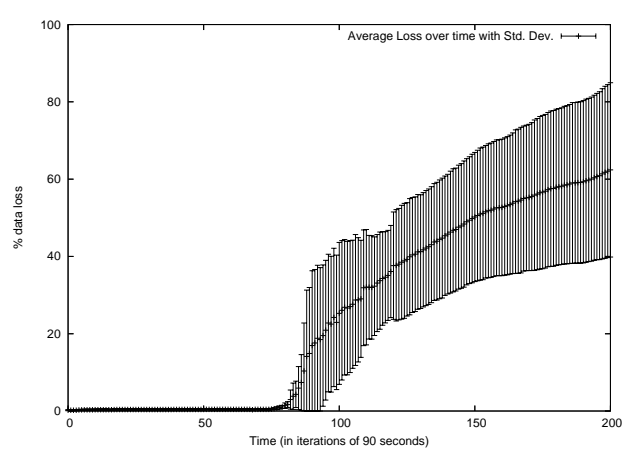

Figure 11. Graph showing average data loss over 5 runs with 800 total nodes and 8 attack nodes using 8 bad locations with the attack starting after about $2 \mathrm{~h}$. 
The attack is initiated after 75 iterations of ordinary network operation. After just 200 iterations (an iteration corresponds to 90 s of Freenet 0.7 operations) the network has lost on average between $15 \%$ and $60 \%$ of its content, depending on the number of attackers. Note that in our model, an individual attacker is granted precisely the same resources as any ordinary user. The figures show the average data loss (and standard deviations) over five runs. For each run, the positions of the attackers were chosen randomly among the 800 nodes.

\subsection{Simulation of Churn}

Figures 12, 13, and 14 show the results of a simulation of join-leave churn on the distribution of node locations in the Freenet network. The total network size was 600 nodes, out of which 500 are stable. In each round, ten of the remaining hundred nodes drop out of the network and join as fresh nodes with a new random location and randomly chosen connections. During each round, the network performs the swap protocol corresponding to about 400ms of a real Freenet network. The experiment was done with various different topologies with similar results. The figures show the results for a topology where the 500 stable nodes are randomly chosen from a small world network, that is, they are not better connected than any of nodes experiencing churn. In all of our simulations, the locations rapidly converge towards a small set of possible locations.

\section{Discussion}

Various strategies could be used to limit the impact of the proposed attack, including changing the swapping policy, malicious node detection, and secure multiparty computation. While some of these strategies can reduce the impact of the attack, we do not believe that adopting any of the suggested measures would address the attack in a satisfactory manner.

One possibility for reducing the effect of the attack proposed in this paper is to increase the amount of time between attempts to swap, or to have each node in the network periodically reset its location to a random value. The idea is that the malicious node locations would spread more slowly and be eventually discarded. However, while this limits the impact of the attack, this defense also slows and limits the progress of the network converging to the most fortuitous topology.

However, the negative impact of churn may be handled by swapping locations only with long lived peers. Recent measurement studies in peer-to-peer networks have shown a power-law distribution of the uptime of peers; a large percentage of peers have a short uptime [16]. By adjusting the probability of location swapping to be proportional to the

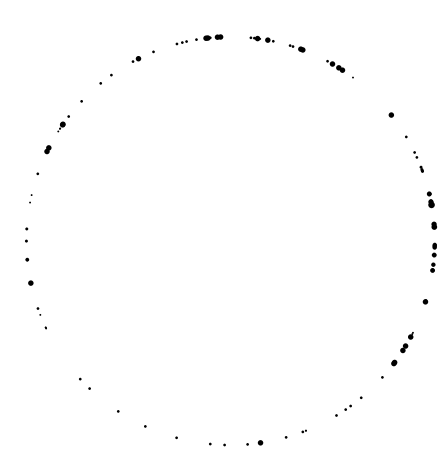

Figure 12. Initial (random) node locations before simulation of join-leave churn.

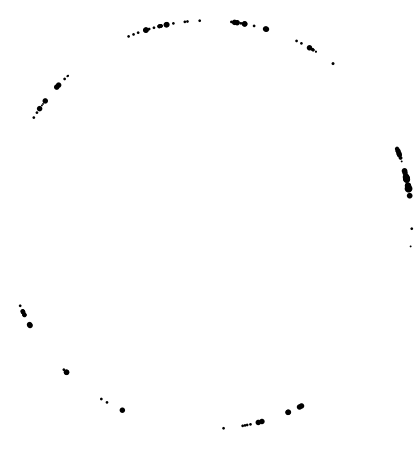

Figure 13. Distribution of the node locations of the stable core of 500 nodes after 100 rounds of churn.

\author{
Figure 14. Distributions of the node locations \\ of the stable core of 500 nodes after 100,000 \\ rounds of churn.
}


uptime of both peers, the network may be able to reduce clustering of the locations of long-lived peers due to joinleave churn.

Another possible method attempts to detect a malicious node based on knowing the size of the network. If a Freenet node were able to accurately produce a close estimation of the size of the network, it could detect if an attacker was swapping locations that are significantly closer than what would be likely with a random distribution of locations. The problem with this approach is that in an open F2F network it is difficult to reliably estimate the network's size.

If there were a way for a node which purported to have a certain number of friends to prove that all those friends existed, nodes could be more confident about swapping. The Freenet developers suggested using a secure multiparty computation as a way for a node to prove that it has $n$ connections. The idea would be for the swapping peers to exchange the the results of a computation that could only be performed by their respective neighbors. But because nodes can only directly communicate with their peers (F2F), any such computation could easily be faked given appropriate computational resources. Of course, if a node could directly communicate with another node's neighbors, then the topology could be discerned. However, in that case the protocol no longer works for restricted-route networks.

\section{Conclusion}

The new Freenet routing algorithm is unable to provide performance guarantees in networks where adversaries are able to participate. The algorithm also degenerates over time (even without active adversaries) if the network experiences churn. The recommended approach to address both problems is to periodically reset the locations of peers. While this limits the deterioration of the routes through adversaries and churn, such resets also sacrifice the potential convergence towards highly efficient routes. Secure and efficient routing in restricted route networks remains an open problem.

\section{Acknowledgements}

The authors thank Ian Clarke, Nils Durner, Krista Grothoff, Oskar Sandberg, Ramakrishna Thurimella and Matthew Toseland for feedback on an earlier draft of the paper.

\section{References}

[1] M. Casado and M. J. Freedman. Illuminating the shadows: Opportunistic network and web measurment. http://illuminati.coralcdn.org/stats/, December 2006.

[2] I. Clarke. The freenet project. http://freenetproject.org/, 2007.
[3] I. Clarke, O. Sandberg, B. Wiley, and T. W. Hong. Freenet: A distributed anonymous information storage and retrieval system. In Proc. of the ICSI Workshop on Design Issues in Anonymity and Unobservability. International Computer Science Institute, 2000.

[4] F. Dabek, M. F. Kaashoek, D. Karger, R. Morris, and I. Stoica. Wide-area cooperative storage with CFS. In Proceedings of the 18th ACM Symposium on Operating Systems Principles (SOSP '01), Chateau Lake Louise, Banff, Canada, October 2001.

[5] M. T. Goodrich, M. J. Nelson, and J. Z. Sun. The rainbow skip graph: a fault-tolerant constant-degree distributed data structure. In SODA '06: Proceedings of the seventeenth annual ACM-SIAM symposium on Discrete algorithm, pages 384-393, New York, NY, USA, 2006. ACM Press.

[6] J. M. Kleinberg. Navigation in a small world. Nature, 406(6798):845-845, August 2000.

[7] J. M. Kleinberg. The small-world phenomenon: an algorithm perspective. In STOC '00: Proceedings of the thirtysecond annual ACM symposium on Theory of computing, pages 163-170, New York, NY, USA, 2000. ACM Press.

[8] P. Maymounkov and D. Mazières. Kademlia: A peer-to-peer information system based on the xor metric. In Proceedings of IPTPS02, Cambridge, March 2002.

[9] S. Milgram. The small-world problem. Psychology Today, pages 60-67, 1967.

[10] W. Pugh. Skip lists: A probabilistic alternative to balanced trees. In Workshop on Algorithms and Data Structures, pages 437-449, 1989.

[11] S. Ratnasamy, P. Francis, M. Handley, R. Karp, and S. Shenker. A scalable content addressable network. Technical Report TR-00-010, Berkeley, Berkeley, CA, 2000.

[12] A. Rowstron and P. Druschel. Pastry: Scalable, Decentralized Object Location, and Routing for Large-Scale Peer-toPeer Systems. Lecture Notes in Computer Science, 2218, 2001.

[13] O. Sandberg. Searching in a Small World. PhD thesis, University of Gothenburg and Chalmers Technical University, 2005.

[14] O. Sandberg. Distributed routing in small-world networks. In ALENEX, 2006.

[15] I. Stoica, R. Morris, D. Karger, M. F. Kaashoek, and H. Balakrishnan. Chord: A scalable peer-to-peer lookup service for internet applications. In Proceedings of the 2001 conference on applications, technologies, architectures, and protocols for computer communications, pages 149-160. ACM Press, 2001.

[16] D. Stutzbach and R. Rejaie. Understanding churn in peerto-peer networks. In IMC '06: Proceedings of the 6th ACM SIGCOMM on Internet measurement, pages 189-202, New York, NY, USA, 2006. ACM Press.

[17] D. Watts and S. Strogatz. Collective dynamics of 'smallworld' networks. Nature, 393:440-442, 1998. 